\title{
Effect of absent bilateral radial arteries on the accuracy of continuous real-time noninvasive blood pressure monitoring with the Nexfin ${ }^{\text {TM }}$ system
}

\author{
Natasha Simula, BA D $\cdot$ Rosie Earle, BSc $\cdot$ Himat Vaghadia, MBBS • \\ Andrew Sawka, MD
}

Received: 22 May 2015/ Accepted: 2 June 2015 / Published online: 6 June 2015

(c) Canadian Anesthesiologists' Society 2015

\section{To the Editor,}

We present a unique case of the use of a Nexfin ${ }^{\mathrm{TM}} \mathrm{HD}$ monitor (BMEYE, Amsterdam, NL) for continuous realtime noninvasive blood pressure (BP) monitoring in a patient with previous bilateral radial artery ligation.

The Nexfin device uses photoplethysmography via a finger cuff to provide noninvasive continuous monitoring of BP, stroke volume, heart rate (HR), and cardiac output. ${ }^{1}$ The device has been validated as a feasible method for continuous hemodynamic monitoring in the majority of patients undergoing surgery and in the intensive care unit, ${ }^{1,2}$ and it has been found comparable with a traditional BP cuff $^{2}$ and thermodilution monitoring. ${ }^{3}$ While this device cannot completely replace the gold standard of invasive intraarterial monitoring, ${ }^{1,4}$ it may still be a suitable option for patients with increased risk factors with radial artery cannulation or where invasive techniques are not warranted or practical. ${ }^{1,3}$ The pulse pressure method used by the Nexfin device relies on an optimal arterial pressure tracing, and any physiologic or artifact interference may cause inaccuracies in Nexfin readings. ${ }^{3}$ The Nexfin device has limited applicability in critically ill patients with poor hand perfusion due to systemic hypotension, use of systemic vasopressors, hypothermia, or severe peripheral edema. ${ }^{1,4}$ Nevertheless, reports are lacking that describe the performance of the Nexfin device in patients with absent radial arteries.

N. Simula, BA $(\bowtie) \cdot$ R. Earle, BSc $\cdot$ H. Vaghadia, MBBS .

A. Sawka, MD

Department of Anesthesia, Vancouver General Hospital, University of British Columbia, Vancouver, BC, Canada e-mail:nsimula@alumni.ubc.ca
We report the case of a 69-yr-old male, American Society of Anesthesiologists physical status III, undergoing right femoral-femoral bypass graft and extended profundoplasty for lower limb ischemia due to peripheral vascular disease. The patient's medical history inlcuded coronary artery disease with coronary artery bypass graft (CABG) surgery 13 years prior as well as multiple previous peripheral revascularization procedures. He had both radial arteries harvested for use as vascular conduits for his CABG surgery. He also had a history of hypertension, type 2 diabetes mellitus, and a 7.5 pack-year smoking history. During the preoperative evaluation, both of the patient's hands appeared well perfused and ulnar pulses were present bilaterally. An Allen's test was not performed. We elected to proceed with femoral-femoral artery bypass under spinal anesthesia. In addition to standard noninvasive monitors, including a brachial BP cuff on the left arm, a Nexfin cuff was placed on the patient's right index finger. Baseline brachial noninvasive BP was measured on both of his arms to ensure there was no pre-existing difference in BP. After a successful subarachnoid block, the surgery proceeded uneventfully.

During the procedure, the mean systolic and diastolic BP readings by traditional $\mathrm{BP}$ cuff monitoring were found to be approximately $165 \mathrm{mmHg}$ and $72 \mathrm{mmHg}$, respectively, compared with $111 \mathrm{mmHg}$ and $46 \mathrm{mmHg}$, respectively, using the Nexfin monitor (Figure). This discrepancy in systolic and diastolic BP (approximately $54 \mathrm{mmHg}$ and 26 $\mathrm{mmHg}$, respectively) was consistent throughout monitoring. The patient maintained a HR of $50-60$ beats $\cdot \min ^{-1}$ as measured by both standard and Nexfin monitoring, and he remained hemodynamically stable throughout the procedure and postoperatively until discharge two days later.

Radial artery harvesting has been shown to produce minimal differences in hand circulation up to two years 


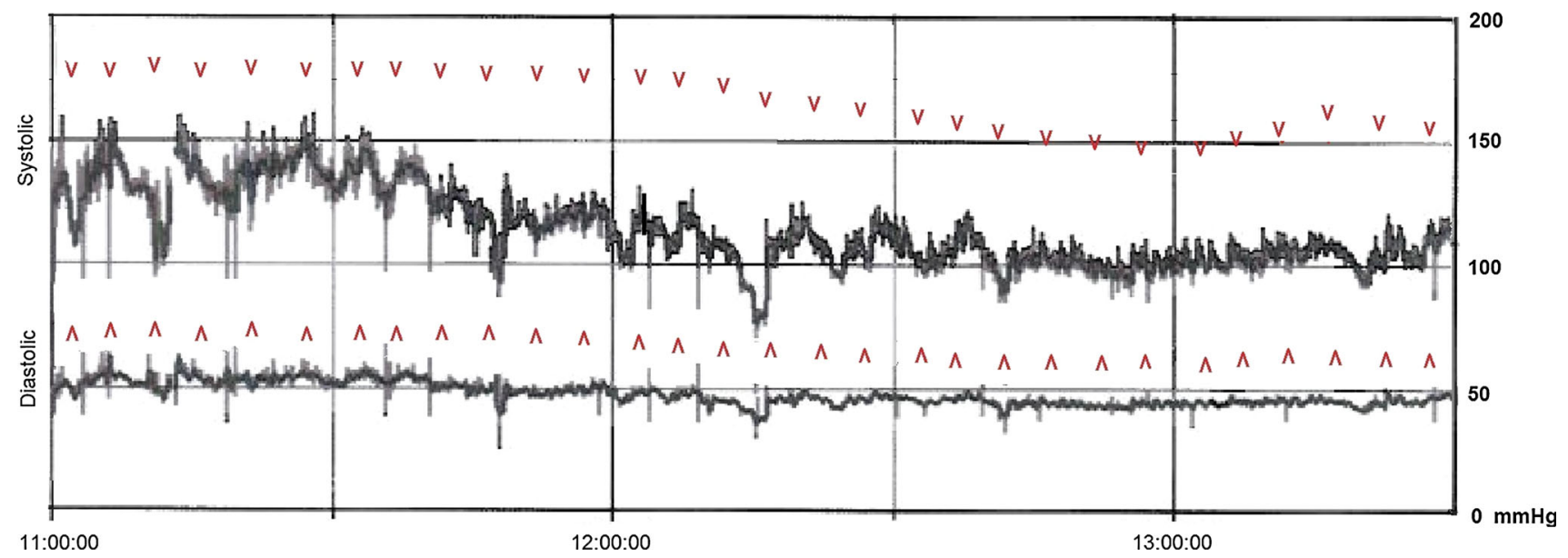

Figure Systolic blood pressure (sBP) and diastolic blood pressure (dBP) from the Nexfin ${ }^{\mathrm{TM}}$ monitor (black) and from the standard intermittent blood pressure cuff (red) during a femoral profundoplasty. The Nexfin reported a mean sBP and dBP of 111

post-procedure due to ulnar compensation. Longer term, however, there can be markedly reduced perfusion due to atherosclerotic changes and intimal thickening. ${ }^{5}$ Our case showed a significant and sustained discrepancy in BP readings between the traditional BP monitor and Nexfin, suggesting that Nexfin is likely not a reliable assessment of $\mathrm{BP}$ in patients with limited or absent radial perfusion. These findings are consistent with previous discrepancies in Nexfin readings found in patients with poor hand perfusion of various etiologies. ${ }^{1,3,4}$

Further research with the Nexfin monitor in patients with limited or absent radial perfusion will help determine whether absent radial arteries should be considered an exclusion criterion for the use of Nexfin for BP monitoring.

Conflicts of interest None declared.
$\mathrm{mmHg}$ and $46 \mathrm{mmHg}$, respectively, while standard monitors reported $165 \mathrm{mmHg}$ and $72 \mathrm{mmHg}$, respectively. This resulted in an average discrepancy of $54 \mathrm{mmHg}$ in $\mathrm{sBP}$ and $26 \mathrm{mmHg}$ in $\mathrm{dBP}$.

\section{References}

1. Ameloot $K$, Palmers PJ, Malbrain ML. The accuracy of noninvasive cardiac output and pressure measurements with finger cuff: a concise review. Curr Opin Crit Care 2015; 21: 232-9.

2. Vos JJ, Poterman M, Mooyaart EA, et al. Comparison of continuous non-invasive finger arterial pressure monitoring with conventional intermittent automated arm arterial pressure measurement in patients under general anaesthesia. Br J Anaesth 2014; 113: 67-74.

3. Thiele $R H$, Bartels $K$, Gan TJ. Inter-device differences in monitoring for goal-directed fluid therapy. Can J Anesth 2015; 62: 169-81.

4. Hohn A, Defosse JM, Becker S, Steffen C, Wappler F, Sakka SG. Non-invasive continuous arterial pressure monitoring with Nexfin does not sufficiently replace invasive measurements in critically ill patients. Br J Anaesth 2013; 111: 178-84.

5. Chim H, Bakri K, Moran SL. Complications related to radial artery occlusion, radial artery harvest, and arterial lines. Hand Clin 2015; 31: $93-100$. 